\title{
Detection and quantification of trends in time series of significant wave heights: an application in the Mediterranean Sea
}

Francesco De Leo ${ }^{1}$, Annalisa De Leo ${ }^{1}$, Giovanni Besio ${ }^{1}$, Riccardo Briganti ${ }^{2}$

\section{Abstract}

The analysis of long-term trends in time series of wave parameters is useful, as these may affect the high return period estimates that are used for the design of several off-shore and onshore engineering projects. This work analyses the use of linear regressions for detecting and quantifying long-term trends in time series of data. In particular, it is evaluated the reliability of a linear trend slope estimate, modified in order to minimize the weight of possible outliers. To this end, this slope is compared against the outcomes of two methods that do not imply the hypothesis of linear trend: the Mann-Kendall test and the Innovative Trend Analysis. An application to significant wave height time series over the Mediterranean Sea is presented. Time series of 40 years of numerical hindcast of sea states over the whole basin were analysed, and the methodology presented was applied to the annual maxima, the annual $98^{\text {th }}$ percentile and the annual mean significant wave height. The results proved that the use of the investigated linear slope is meaningful and

\footnotetext{
${ }^{1}$ Department of Civil, Chemical and Environmental Engineering, University of Genoa, Genoa, 16145, Italy

${ }^{2}$ Faculty of Engineering, University of Nottingham, Nottingham, NG7 2RD, United Kingdom
} 
sound, therefore this was used to assess the spatial distribution of trends in the Mediterranean Sea. Results are presented and discussed for all the statistics investigated.

Keywords: long-term trends, time series analysis, Mediterranean Sea

\section{Introduction}

Climate change is expected to significantly affects the main met-ocean parameters, at both large scale and the local scale [50]. Relevant changes are taking place in the upper-sea physics, and in particular in water temperature and salinity [13], large-scale circulation [5, 4], mean sea level [30], and wave heights and periods $[46,27]$. In view of these considerations, the evaluation and prediction of the upper-sea physics trends plays a crucial role in a plethora of geophysical studies and engineering applications, such as the erosion of the coasts [43], changing design from hard to soft engineering options [17], flooding hazard and coastal vulnerability assessment and management $[1,35,11,49]$, marine ecosystems $[18,33,12]$. The present study focuses on the variations in wave climate, in particular in trends of significant wave height (henceforth $H_{s}$ ); this is an issue of primary concern, because these may affect the fluxes of energy between the ocean and the atmosphere and even storm surges [51]. These variations would be also important for the coastal areas, as they may in turn modify the equilibrium conditions of coastal beach profiles [26] and affect ports' activity to a substantial extent [20]. It is therefore crucial to identify and quantify the trends in wave climate, to embed these information in engineering design.

The simplest approach to quantify a trend in sea state datasets is to 
perform a linear regression over the values of the time series. As an instance, [16] applied a linear least square regression to annual mean $H_{s}$ observed from ship routes over the last century on a global scale. The same approach was used by [39] to analyse both the annual mean and $90^{\text {th }}$ percentile of $H_{s}$ in the Central Bay of Bengal, and by [29] in the Eastern Mediterranean, while [2] performed linear interpolations of monthly wave height statistics in the Gulf of Mexico. Linear slopes estimates were also employed to characterize extremes $H_{s}$ selected with the Peak Over Threshold (POT) analysis in the Italian seas [31], along the Catalan coast [6] and along the Chinese coast [40].

Previous researches made also use of linear regression modified according to the model of Theil-Sen $[36,44]$, resulting in a sounder slope estimate (hereinafter TS slope), because it is insensitive to possible outliers. The TS slope was used, among others, by [32], evaluating monthly quantiles trends in the northern Adriatic Sea, in [51], assessing $H_{s}$ global trends for annual mean, mode and $90^{\text {th }}$ percentile, and in [47], who compared the TS slopes with four different models for detecting long-term trends of $H_{s}$ in the North Atlantic. In particular, [47] employed the seasonal ARIMA (AutoRegressive Integrated Moving Average) modelling, multiple regression modelling, and GAM (Generalized Additive Model) modelling, and showed that the different approaches result in reasonable agreement. Beside [47], other works performed trend analysis characterized by major complexity than the linear regression over time series of $H_{s}$; among others, [8] used a wavelet analysis for assessing the variation in time of the dominant temporal modes of variability in the Atlantic coast of Europe; [28] modelled historical trends of extreme $H_{s}$ in two Portuguese locations through regression quantile models. 
In most of the applications that aim to evaluate changes in geophysical time series, the identification of trends is usually carried out using the nonparametric Mann-Kendall statistical test [23, 19, hereinafter called MK], based on the samples rank correlation within a dataset. The MK, as well as many other statistical tests, allows to accept or reject the hypothesis it verifies (the so called null hypothesis, in this case the absence of a climate trend) on the basis of the variable $p_{\text {value }}$, defined as the observed significance level for the test hypothesis. The $p_{\text {value }}$ is compared with a significance level $\alpha$, used as a threshold, to reject (if $p_{\text {value }}<\alpha$ ) or accept (if $p_{\text {value }} \geq \alpha$ ) the null hypothesis. In its common use, the MK does not provides any information on the trend magnitude. In the context of trends of $H_{s}$, the MK was employed, for example, in the aforementioned studies by [6] and [32], who selected a threshold of $\alpha=0.1$ to identify and subsequently characterized locations showing trends off the Catalan coast and in the Adriatic sea, respectively. Similarly, [2] and [40] performed linear interpolations on $H_{s}$ time series for locations showing trends at a level of $\alpha$ (i.e., threshold level) equal to 0.05 .

Nevertheless, it should be mentioned that there is no theoretical basis for the definition of the threshold value $\alpha$, for that the binary use of $p_{\text {value }}$ has been increasingly questioned over the last few years. According to [48] and [15], the $p_{\text {value }}$ should be considered as a continuous measure spanning the 0-1 range; 1 indicates that data behave consistently with the null hypothesis, while values tending to zero indicate that data behave progressively less consistently with the null hypothesis. In view of the above, the $p_{\text {value }}$ of MK (referred to as $p_{M K}$ ) can be used as a measure of compatibility between the data and the hypothesis that they are not characterized by a long-term 
trend. A similar use of $p_{\text {value }}$ is found in [42], where the $p_{\text {value }}$ of the AndersonDarling statistic was used as a goodness-of-fit measure, to check whether their data were best represented by a Generalized Pareto Distribution. In case of trend analysis, one further limitation of the traditional use of the MK is that a value of $\alpha$ is required to evaluate the sign of a trend. On the contrary, the slope of the best fitting line immediately reveals whether the data of a series are most likely to increase (positive slopes) or decrease (negative slopes) in time. Indeed, the main advantage related to the use of linear slope estimates, is that they provide easy-to-read and prompt information of long-term trends over time series of data, with respect to more complex models which may be difficult to read for many analysts. However, the hypothesis of linear rate of change may represent a too limiting assumption.

In this paper, we evaluate whether the TS slope can be efficiently employed to quantify the sign and the magnitude of a trend, even if the underlying trend is not linear. To this end, we take advantage of hindcast data defined over the Mediterranean Sea (MS), computing the annual maxima, the annual mean and the annual $98^{\text {th }}$ percentile of $H_{s}$ over the whole basin. First, we investigate how the TS slopes of the reference time series relate to their respective $p_{M K}$; indeed, MK does not postulate the linearity of the underlying trend. Subsequently, we compare the TS estimates with the outcomes of another method that is not bounded by the hypothesis of linear trend [the so called Innovative Trend Analysis, hereinafter referred to as ITA, 37, 38]. Finally, once the suitability of the TS slope for detecting long-term trends is proved, we evaluate the spatial distribution of long-term trends of the extremes and the mean $H_{s}$ over the MS. 
The paper is structured as follows: in Section 2 we present the hindcast data used for the study, along with the methodologies adopted to detect climate trends throughout the MS and the correlations analysis employed for linking the TS slopes with the methodologies against which they are evaluated. Section 3 shows and discusses the results of the correlation analysis and a regional overview of the trends distributions over the MS. Finally, results are further summarized in Section 4.

\section{Data and Methods}

\subsection{Wave hindcast and selection of data}

Wave data used here were computed by the hindcast service of the Department of Civil, Chemical and Environmental Engineering of the University of Genoa [24, 25]. The service provides the main wave features on a hourly basis over a 40 years long period (from January 1979 to December 2018 ), with a $0.1273^{\circ} \times 0.09^{\circ}$ lon/lat spatial resolution (side of the cells of the computational grid is of the order of $10 \mathrm{~km}$ at the latitude of $45^{\circ} \mathrm{N}$ ) over the whole MS. Generation and propagation of sea waves are modelled using WavewatchIII ${ }^{\circledR}$ version 3.14 [45], forced by means of the nonhydrostatic model Weather Research and Forecasting-Advanced Research 3.3.1 [WRF-ARW, 41], based on the Climate Forecast System Reanalysis database [CFSR, 34]. This dataset has been already used for a number of studies on storms and wave climate over the Mediterranean Sea [3, 9, among others].

In order to detect trends in extreme sea state time series, the events considered to be extremes were extracted from the whole time series of $H_{s}$ under 
study. In the extreme value analysis framework, the POT has become a wellsettled methodology, often preferred to the Annual Maxima (AM) approach, above all for relatively short time series. However, the POT requires to select a $H_{s}$ threshold that may significantly affect the subsequent trend analysis, either in terms of magnitude and number of resultant peaks [21, 22]. The value of the threshold may also be affected by climatic trends, e.g. if the $H_{s}$ corresponding to the $98^{t h}$ percentile is taken as threshold for the POT, this value will vary in time in presence of a trend. Additionally, the number of events above a given threshold varies every year and different nodes in the grid considered might have different number of events per year, posing additional problems of homogeneity of the reference population, with respect to AM values. On the basis of these considerations, the AM $H_{s}$, annual $98^{\text {th }}$ percentile of $H_{s}$ and annual mean $H_{s}$ were chosen for the analysis, assuring that one sample per year is used across the grid in all cases.

\subsection{Trend detection and quantification}

\subsubsection{TS method}

The simplest trend is a linear one, hence, in order to quantify it, it is possible to use the slope of the linear fit of a series of data. The value of this slope can be computed following the TS method that is insensitive to outliers, and it is therefore preferred to other common tools, such as the least squares regression, for the problem under study. Considering a series of values $x_{i}(i=1 \ldots n, n$ being the number of samples) the estimate of the TS slope $(b)$ is computed as: 


$$
b=\operatorname{Median}\left(\frac{x_{j}-x_{l}}{j-l}\right) \forall l<j, l, j=1 \ldots n
$$

143

where $x_{j}$ and $x_{l}$ are the $j^{\text {th }}$ and $l^{\text {th }}$ data of the series, respectively.

\subsubsection{The Mann-Kendall test}

The MK is aimed at evaluating whether an either upward or downward monotonic trend is present within a dataset. The null hypothesis of the test is that there is no monotonic trend in the time series. The test statistic $Z_{M K}$, considering a time series of $n$ elements $x_{i}, i=1 \ldots n$, is computed as:

$$
Z_{M K}=\frac{\text { num }}{\sqrt{\sigma^{2}(S)}}
$$

where num is equal to:

$$
\operatorname{num}=\left\{\begin{array}{l}
S-1, \text { if } S>0 \\
0, \text { if } S=0 \\
S+1, \text { if } S<0
\end{array},\right.
$$

and $S$ and $\sigma^{2}(S)$ are computed as:

$$
\begin{aligned}
S & =\sum_{k-1}^{n-1} \sum_{j-k+1}^{n} \delta_{j-k} \\
\sigma^{2}(S) & =\frac{1}{18}\left[n(n-1)(2 n+5)-\sum_{p-1}^{g} t_{p}\left(t_{p}-1\right)\left(2 t_{p}+5\right)\right]
\end{aligned}
$$

with $\delta_{j-k}$ being an indicator function that takes 1,0 or -1 value according to the sign of $x_{j}-x_{k}$ (positive, null or negative, respectively); $g$ is the number of tied groups in the time series, with $t_{p}$ being the number of elements in each 
$p^{t h}$ group $(p=1,2, . . g)$. The value of $Z_{M K}$ is then evaluated as a percentile of the standard normal distribution, leading to the corresponding $p_{M K}$ of the statistic. In most of the applications that take advantage of the MK, the $p_{M K}$ is successively compared to $\alpha$. In such a case, the use of $\alpha$ also allows to detect the sign of the trend (whether it is upward or downward oriented) using the following relationships:

$$
\left\{\begin{array}{l}
Z_{M K}>\phi^{-1}(1-\alpha / 2) \rightarrow \text { positive trend } \\
Z_{M K}<-\phi^{-1}(1-\alpha / 2) \rightarrow \text { negative trend } \\
Z_{M K}<\left|\phi^{-1}(1-\alpha / 2)\right| \rightarrow \text { no trend }
\end{array},\right.
$$

where $\phi$ stands for the transform in the normal space.

Nevertheless, in this research the $p_{M K}$ of each annual statistic was evaluated in the whole 0-1 range. In this way, $p_{M K}$ was used to assess the intensity of trends over the wave height series, as previously explained in Sect. 1.

\subsubsection{Innovative Trend Analysis}

The approach known as ITA is here briefly introduced. This method requires to split a series in two halves, each with elements sorted in ascending order, and plotted versus each other in a square plot. This allows to evaluate how the scatters diverge from the bisecting line, which represents the notrend condition. Therefore, the ITA allows to quickly check for increasing or decreasing trends (whether the scatter lies above or below the bisector respectively). An example can be seen in Figure 1, in which three realizations of generic time series $\left(x_{1}\right.$ and $\left.x_{2}\right)$ are first ordered according to the ITA procedure and then plotted against each other. 


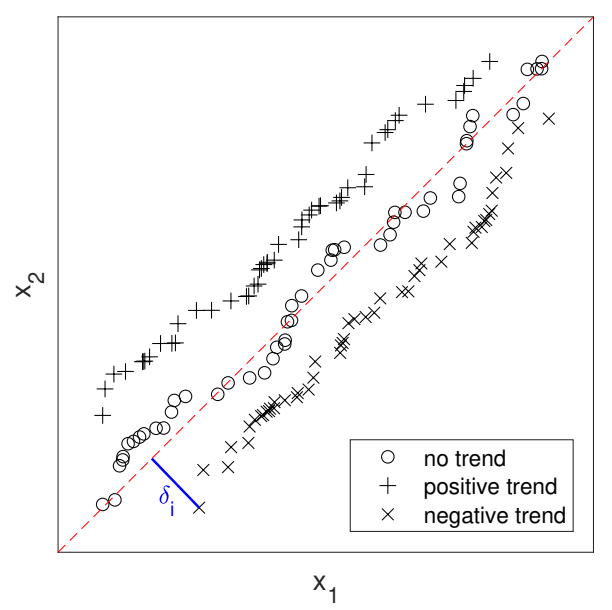

Figure 1: ITA plot for datasets characterized by positive (black crosses), negative (black $\mathrm{x})$ and no trend (black circles)

$\delta_{i}$ is the distance between the $i^{t h}(1 \ldots n / 2)$ element of the series and the no-trend line (see Figure 1). Consequently, for each wave height dataset treated using the ITA, a series of $n / 2 \delta_{i}$ can be built (where $n$, in the present analysis, is the number of years over which the hindcast data are defined). Note that the sign of $\delta_{i}$ indicates that the $i^{\text {th }}$ value lays below $\left(\delta_{i}<0\right)$ or above $\left(\delta_{i}>0\right)$ the no-trend line. If $\delta_{i}>0$ the trend is positive, and it is negative for $\delta_{i}<0$, while change in sign of $\delta_{i}$ indicates that not all data behave consistently with the presence of a trend.

\subsection{Analysis of the correlation between the variables employed}

The proposed methodology makes it possible to combine $b$ and $p_{M K}$ without restriction for the rejection of the null hypothesis. Subsequently, $b$ is shown to be correlated with the parameters of the population of $\delta_{i}$ in the ITA method. 


\subsubsection{Analysis of the correlation between $b$ and $p_{M K}$}

The correlation between $p_{M K}$ and $b$ was analysed for all the hindcast locations following [14]. Correlations were graphically evaluated in the unitsquare space, spanning the $0-1$ range and populated by the scaled ranks $\left(S R_{i}\right)$ of the investigated variables,

$$
S R_{i}=\frac{m_{i}}{n+1}
$$

where $m_{i}$ is the position of the $\mathrm{i}^{\text {th }}$ data within the sorted series it belongs to, whereas $n$ in our case equals the number of years covered by the hindcast. The scatter plot of ranks of $p_{M K}$ versus ranks of $b$ is a visual tool that indicates the presence of correlation, anti-correlation, or no correlation at all. In the case of correlation, high (low) ranks of $p_{M K}$ occurr frequently together with high (low) ranks of $b$. In the case of anti-correlation, high (low) ranks of either variables tend to occur with low (high) ranks of the other. No correlation is characterized by the absence of either of the previous patterns $[14]$.

Correlation levels were then quantified through the Spearman grade correlation coefficient $\left(\rho_{s}\right)$. Said $R_{p . i}$ and $R_{b, i}$ the $\mathrm{i}^{\text {th }}$ ranks of $p_{M K}$ and $b$ respectively, the following expression generally applies:

$$
\rho_{s}=\frac{12}{n(n+1)(n-1)} \sum_{i=1}^{n} R_{p, i} R_{b, i}-3 \frac{n+1}{n-1} .
$$

$\rho_{s}$ was selected because it has the advantage of being always defined, unlike other commonly employed correlation coefficients, such as the classical Pearson coefficient, which directly depends on the second-order moments of the variables of interest, that is not always guaranteed [10]. The values of 
$\rho_{s}$ span from -1 (for series perfectly anti-correlated) to 1 (series perfectly correlated); $\rho_{s}$ equal to 0 indicates that no correlation exists between the investigated series. Correlations were iteratively evaluated by varying the significance level $\alpha$ within the 0 - 1 range with a 0.01 incremental step. For every iteration, only the series with $p_{M K}<\alpha$ were retained for the analysis, i.e. just the series allowing to reject the MK null hypothesis according to the binary use of $p_{M K}$. When $\alpha$ equals 1 , no data are excluded and all the hindcast locations are taken into account; indeed, the maximum value that $p_{M K}$ can attain is exactly 1 , therefore in the latter case no filtering on the series due to the value of $\alpha$ is applied.

\subsubsection{Analysis of the correlation between $b$ and the distribution of $\delta_{i}$}

The second step of the developed methodology, requires to check whether the values of $b$ are consistent with the $\delta_{i}$ obtained by the ITA, referred to the respective series (i.e. the $H_{s}$ annual statistics of the hindcast locations).

The reliability of the linear trend hypothesis was first evaluated by analysing the empirical cumulative distribution function (ecdf) of $\delta_{i}$ series for four hindcast locations characterized by different values of $b$. This allows to check rapidly if the $\delta_{i}$ series increase or decrease according to the values of $b$ the ecdf is linked to, meaning that the larger is $b$, the larger are the $\delta_{i}$.

However, a graphical comparison for all the hindcast locations would not be feasible due to the high number of available datasets. Therefore, the sum of the $\delta_{i}$ for each $H_{s}$ time series was computed, therefore using this sum as a single parameter for the analysis instead of $n / 2$ data. This allowed to perform a direct comparison between datasets of equal length (e.g. the number of hindcast locations): one containing the values of $b$ and the other 
with the sum of $\delta_{i}$ (for each of the annual statistics taken into account).

\section{Results}

\subsection{Trend identification and quantification}

First, the correlations between $b$ and $p_{M K}$ for the AM $H_{s}$ are shown in Figure 2. For the sake of clarity, only results related to four levels of $\alpha$ are here reported $(0.01,0.05,0.90$, and 0.95$)$. The panels show as well the values of $\rho_{s}$ computed for the AM $H_{s}$ series showing $p_{M K} \leq \alpha$.
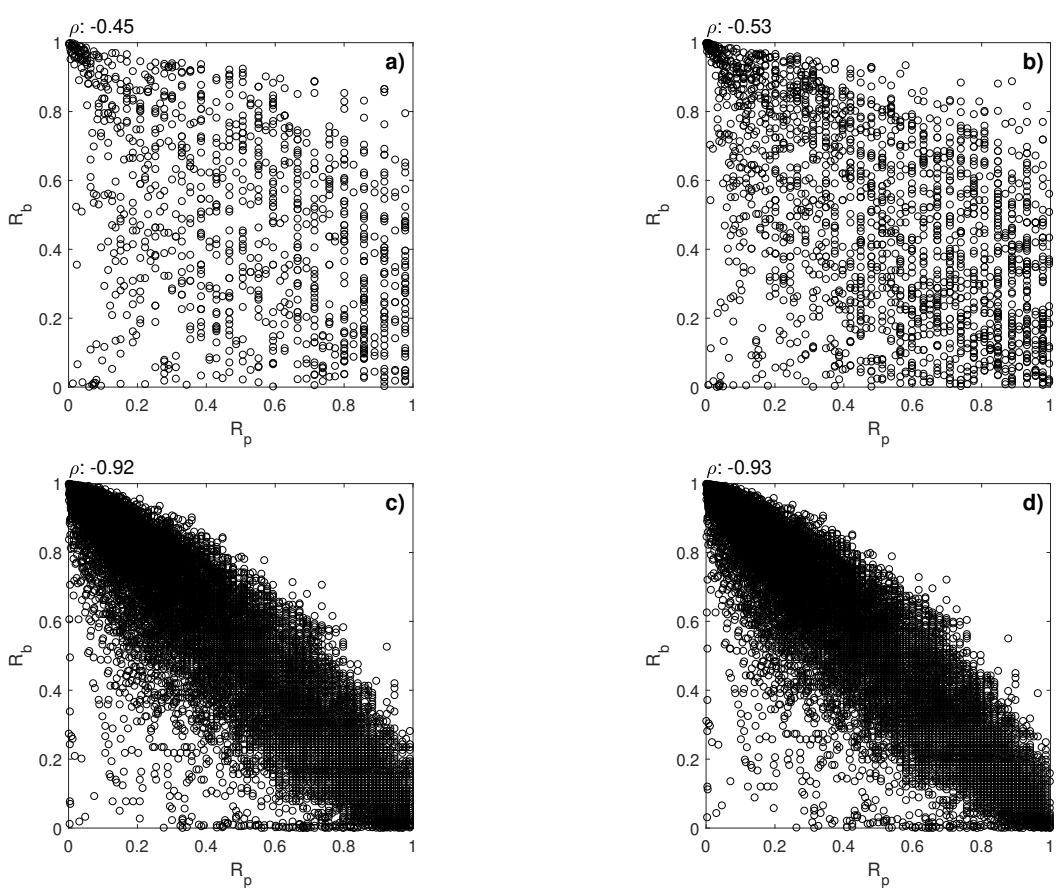

Figure 2: Correlations between $b$ and $p_{M K}$ due to different values of $\alpha$. Panel a): $\alpha=0.05$; panel b): $\alpha=0.1$; panel c): $\alpha=0.9$; panel d): $\alpha=0.95$

The series with $p_{M K}<\alpha, \alpha=0.05 \mid 0.1$ (Figure 2, panels a) and b), show no appreciable correlation between $p_{M K}$ and $b$ can be detected. In fact, the 
scatters of $R_{b}$ and $R_{p}$ are almost randomly distributed over the square-unit space, and the values of the respective $\rho_{s}$ are far from -1, which indicates a perfect anti-correlation. On the other hand, analyzing the correlation for $p_{M K}$ retained considering higher values of $\alpha(0.9 \mid 0.95$, Figure 2 panels $\mathrm{c})$ and d)), show a striking anti-correlation between the two investigated parameters. As shown by the distributions of $R_{p}$ and $R_{b}$ in panels c) and d) of Figure 2, low values of $p_{M K}$ are most likely to occur when $b$ attains high values and vice-versa: the scatters of the scaled ranks are homogeneously distributed along the -1 bisector, and $\rho_{s}$ reaches values close to -1 . As explained in Sect. 2.3.1, the full range of $\alpha$ was explored; Figure 3 shows the results of $\rho_{s}$ as a function of $\alpha$. For the sake of clarity, hereinafter values of $b$ related to the $\mathrm{AM}$, annual $98^{\text {th }}$ percentile and annual mean $H_{s}$ are referred to as $b_{A M}, b_{98}$ and $b_{M E A N}$, respectively. In panel a) of Figure 3 it can be noticed how the anti-correlation between $p_{M K}$ and $b_{A M}$ becomes stronger (i.e. $\rho_{s}$ tends to -1) proportionally to the level of $\alpha$ taken into account. Similar outcomes were found for $b_{98}$ and $b_{M E A N}$; in these cases, only the results of the $\rho_{s}$ series are shown (Figure 3 panel b) and panel c).

As explained in Sect. 2.3.2, the series of $b$ were further compared with the ITA results. To this end, four AM $H_{s}$ series were analyzed; they are characterized by either very intense trends (Point_001337 and Point_005995, upward and downward trend respectively), and by almost flat trends (Point_013330 and Point_021272), according to the values of the respective $b_{A M}$. Then, the ecdf of the respective $\delta_{i}$ were computed. 

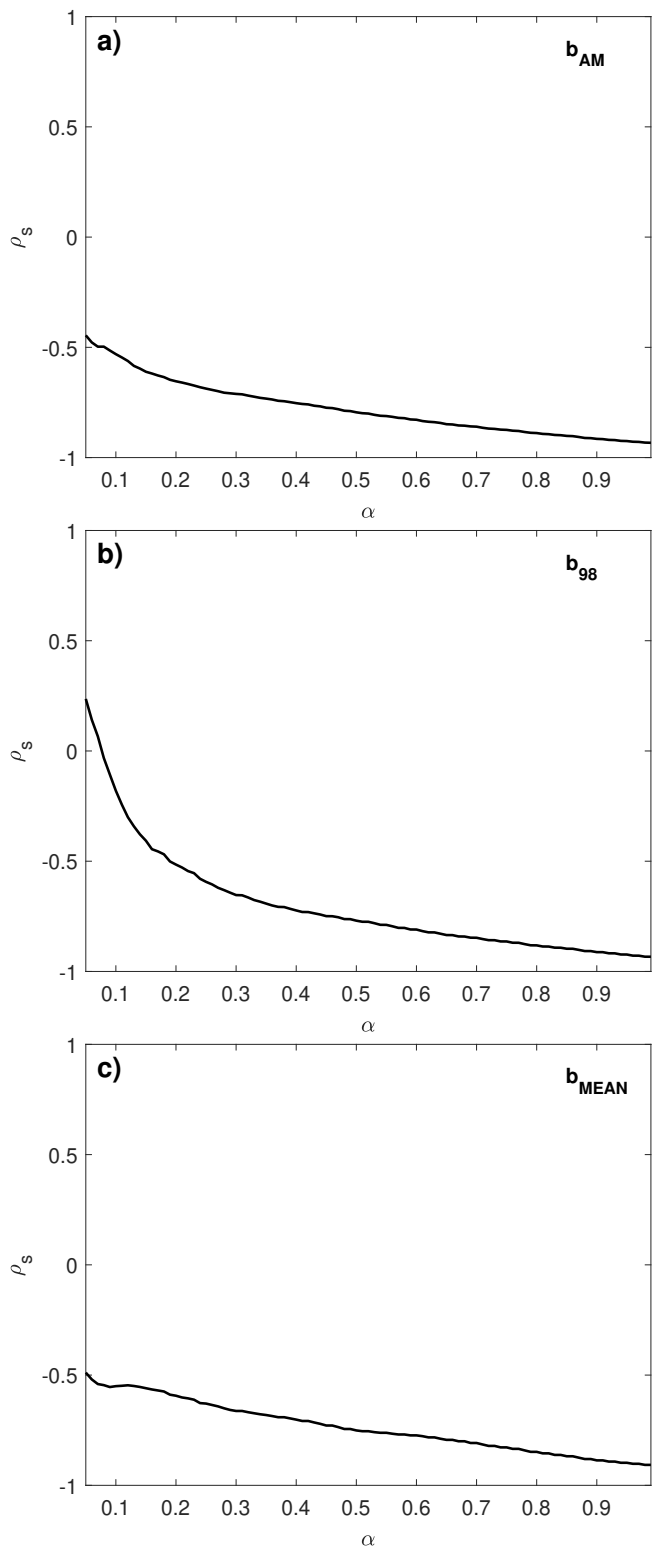

Figure 3: $\rho_{s}$ for the correlation between $b$ and $p_{M K}$ for different values of $\alpha$. Panel a): AM data; panel b): annual $98^{\text {th }}$ percentile of $H_{s}$; panel c): annual mean $H_{s}$ 
The locations of the hindcast points taken into account are shown in Figure 4, while the AM $H_{s}$ series of the selected locations are shown in Figure 5. As Figure 6 shows, the series with almost flat trends (e.g. $b$ close to zero, panels c)-d) of Figure 5) show $\delta_{i}$ with an approximately vertical profile in the ecdf space; on the other hand, series related to steeper trends (panels a)-b) of Figure 5) are characterized by $\delta_{i}$ further shifted from the 0 line. This analysis reveals how the higher (lower) values of $\delta_{i}$ are in turn linked to the higher (lower) values of $b$. This applies both to upward (positive slopes, right half of Figure 6) and downward (negative slopes, left half of Figure 6) trends.

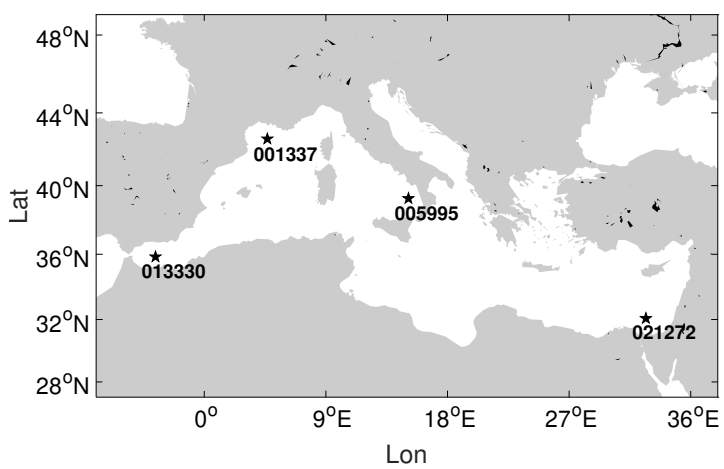

Figure 4: Locations of the hindcast points employed for the graphical comparison between $b$ and $\delta_{i}$

and $b_{M E A N}$ have similar $\rho_{s}$, while the correlation is slightly lower between $b_{98}$ and the respective $\delta_{i}$. 

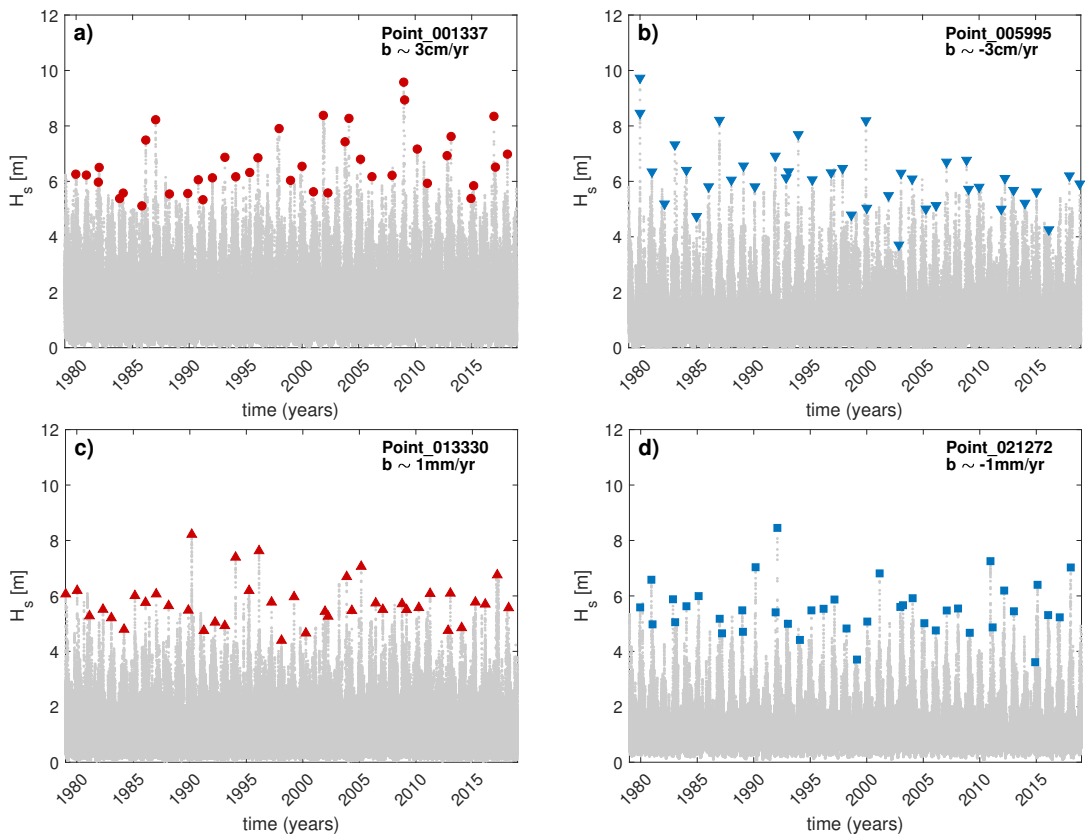

Figure 5: Panels a) and c): AM $H_{s}$ series with respective TS slopes for upward trends. Panels b) and d): downward trends. Red markers: AM $H_{s}$ characterized by positive trends; blue markers: AM $H s$ characterized by negative trends; gray markers: original time series

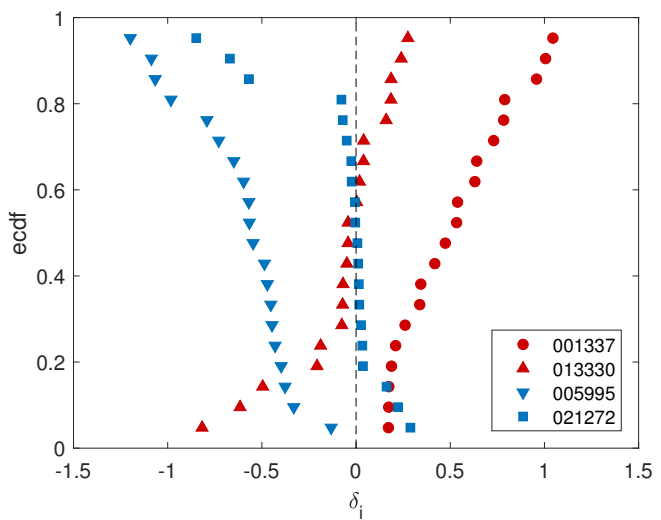

Figure 6: $\delta_{i}$ ecdf for the locations characterized by different trend intensities for AM $H_{s}$ series shown in Figure 5 


\begin{tabular}{cccc}
\hline & $\sum \delta_{i_{A M}}-b_{A M}$ & $\sum \delta_{i_{98}}-b_{98}$ & $\sum \delta_{i_{M E A N}}-b_{M E A N}$ \\
\hline$\rho_{s}$ & 0.79 & 0.67 & 0.85 \\
\hline
\end{tabular}

Table 1: Values of $\rho_{s}$ for the correlation between the series of sums of $\delta_{i}$ and $b$ for the annual $H_{s}$ statistics analyzed

\subsection{Wave climate trends in the Mediterranean Sea}

First, it is interesting to analyse the spatial distribution of $H_{s}$ trends when the most common usage of the MK, relying on the threshold $\alpha=0.05$, is applied. Figure 7 shows only the locations characterized by trends according to the aforementioned method for the AM data, annual $98^{\text {th }}$ percentile, and annual mean $H_{s}$ (panels a), b) and c) of Figure 7). The sign of the trends is computed using Eq. (6). The $\mathrm{AM} H_{s}$ results show large areas characterized by negative trends in the south Tyrrhenian Sea and in the Ionian Sea, while smaller areas and isolated spots showing positive trends are present, for instance, in south-east of the Aegean Sea and in the northernmost areas of the MS. The results for the annual $98^{t h}$ percentile of $H_{s}$ show positive trends in the south of the MS between Sicily and Libya, while negative trends are limited to very few locations. Results for the annual mean $H_{s}$ show negative trends limited to the south-east basin of the MS and positive trends limited to small spots in front of the Libyan coast and along the coastlines of Italy and Greece. 

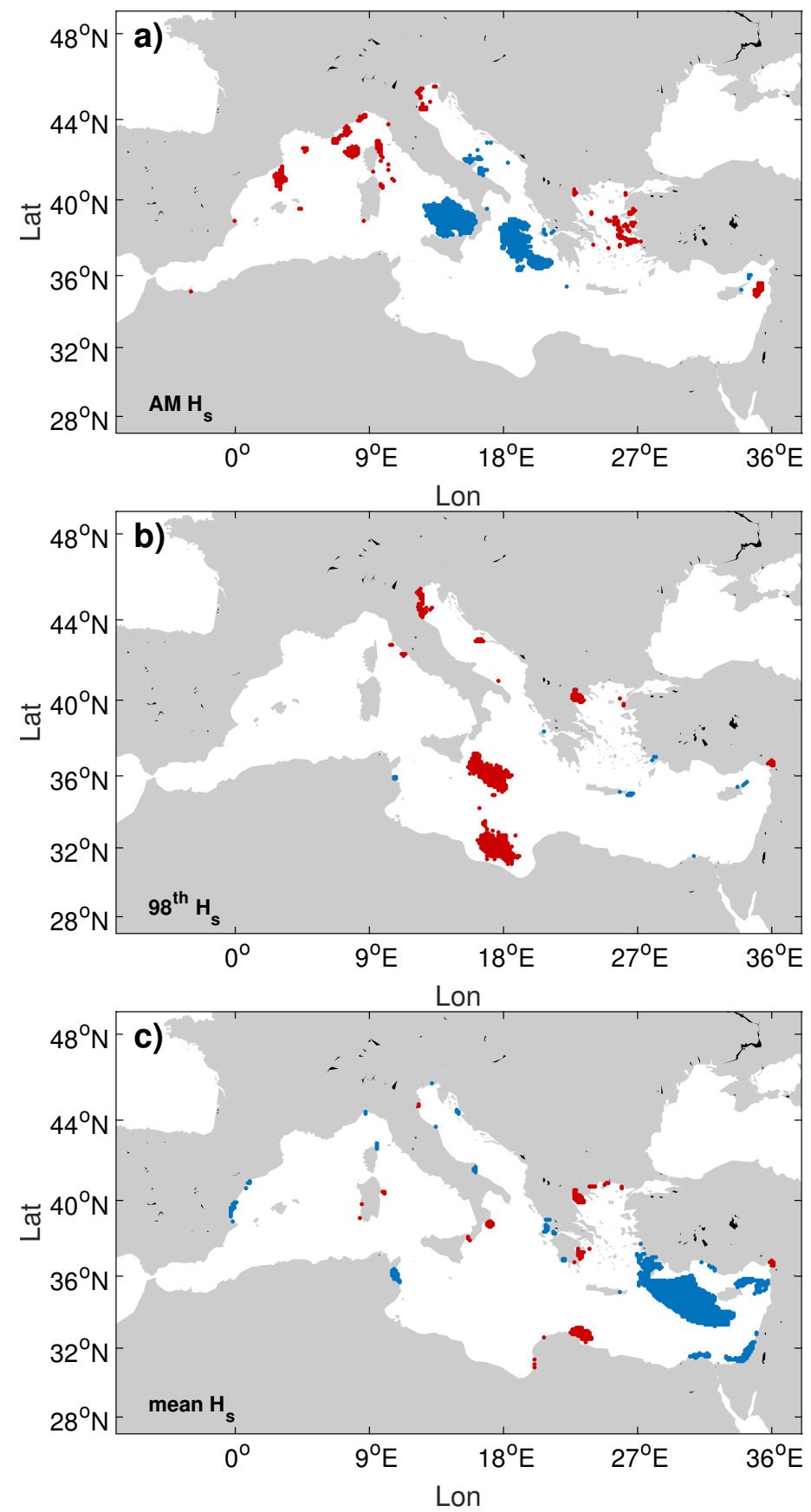

Figure 7: Locations characterized by MK trends for $\alpha$ equal 0.05. Panel a): AM $H_{s}$; panel b): annual $98^{\text {th }}$ percentile of $H_{s}$; panel c): annual mean $H_{s}$. Red dots indicate positive trends, blue dots indicate negative trends 
On the other hand, Figure 8 shows the values of $b$ computed for the annual statistics of $H_{s}$ over the MS, for both downward and upward trends. It can be can seen that the most significant $b$ for the $\mathrm{AM} H_{s}$ are between $-5 \mathrm{~cm} /$ year and $3 \mathrm{~cm} /$ year. The areas subjected to the most intense negative trends are the south of the Tyrrhenian Sea (in front of the northern coasts of Calabria and Sicily) and the Ionian Sea, opposite the Greek coasts. On the other hand, the Aegean Sea and the Tyrrhenian Sea (on east Corsica and Sardinia), together with areas spread within the Balearic Sea, show wide areas subject to positive trends of the AM $H_{s}$. Results for the mean and the $98^{\text {th }}$ percentile of $H_{s}$ change dramatically with respect to those of AM $H_{s}$. The trends show magnitude of mm/year, with different spatial distribution: for both $b_{M E A N}$ and $b_{98}$, areas with negative values can be appreciated in the south-east of the MS and in the north Tyrrhenian Sea, while positive trends are found in the west of Sardinia and in the area between Libya and the Ionian Sea. 

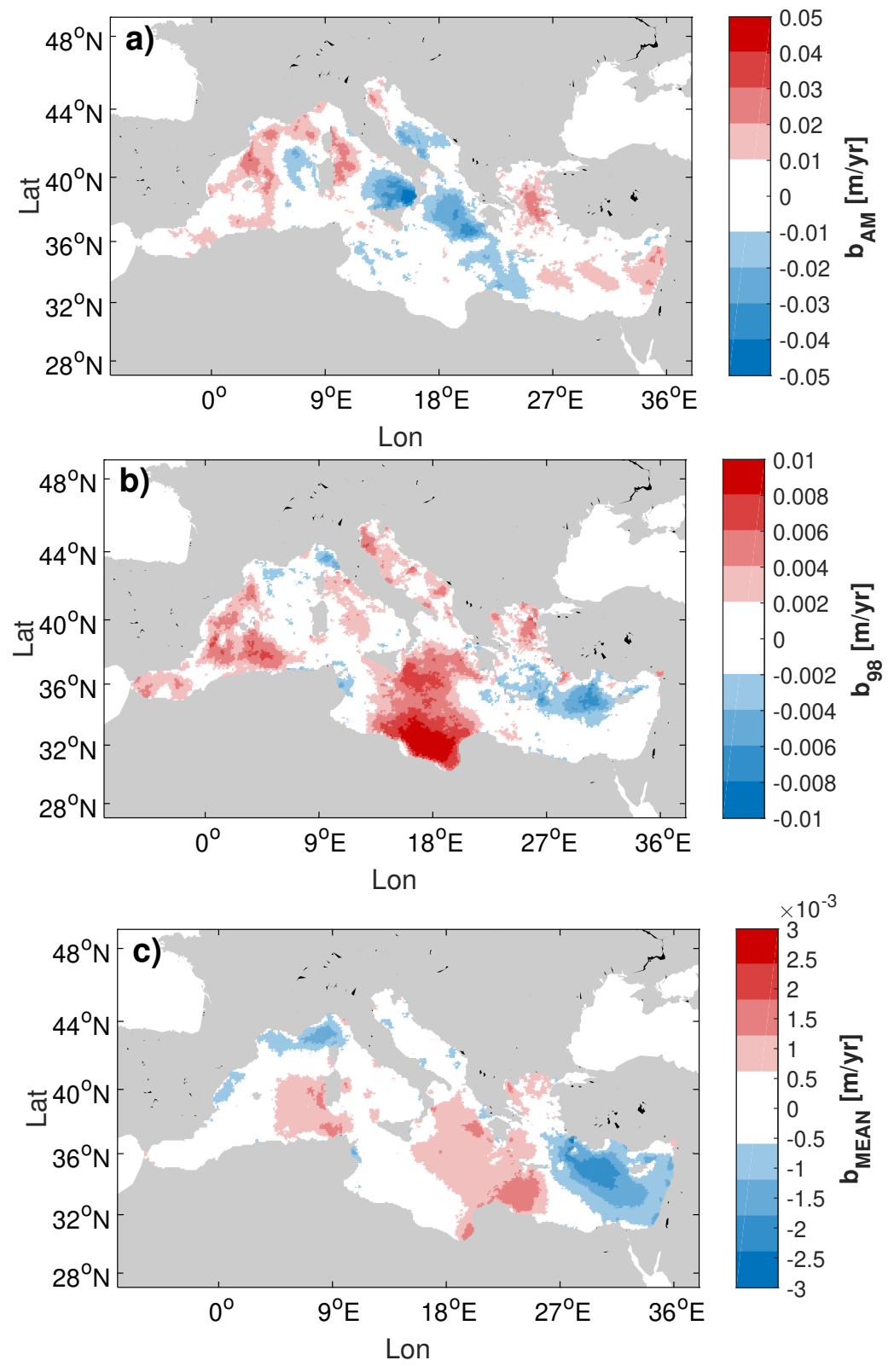

Figure 8: Spatial distribution of $b$ in the MS. From top to bottom: Panel a): $b_{A M}$; panel b): $b_{98}$; panel c): $b_{M E A N}$ 
To the best of our knowledge, it is the first time that an analysis of wave climate trends is performed on the whole MS with such a resolution. Therefore, comparison with previous results can be carried out only considering local analysis in the literature. [6] and [7] evaluated trends for different directional sectors along the Catalan coast; [31] carried out a study on the Italian seas. However, in the aforementioned works trends were computed considering sea storms extracted by de-clustering threshold exceedance within the POT approach, thus a direct comparison with the present analysis would be not significant. [32] evaluated trends on several monthly percentiles of $H_{s}$ in the northern Adriatic Sea, showing a reduction in extremes and an increase in storminess that is not fully consistent with the results of Figure 8, where $b_{98}$ is positive but no trend in AM $H_{s}$ is found. Statistics based on annual intervals in eastern Mediterranean were carried out by [29], further employing a simple linear regression for computing trends; their analysis of annual mean $H_{s}$ returned negative trends of order of magnitude consistent with the present work, however, in their research, no positive trend is identified. As far their AM $H_{s}$ analysis, local analysis within the Aegean Sea agrees qulitatively well with the outcomes of the present work. On the other hand, [29] showed a slightly negative trend in front of the coast of Lebanon, while the present analysis suggests an area subject to homogeneous positive trends. On the contrary, the trend for $90^{\text {th }}$ percentile in the same area is positive and apparently more consistent with the outcomes of the present work for the annual $98^{\text {th }}$ percentile of $H_{s}$. 


\section{Discussion and Conclusions}

In most of the studies that use the MK, $p_{M K}$ is evaluated against a threshold value of $\alpha$ to check for the presence of a trend. In the present work, by using the Spearman index as measure of correlation it was found that for the typical values of $\alpha$ used, the values of $b$ do not appear to be significantly related to the $p_{M K}$ they refer to. Therefore, in this case no useful considerations can be inferred for $b$, regardless the assumptions made about the use of $p_{M K}$. On the contrary, when $p_{M K}$ is considered in its whole range, a clear anti-correlation with $b$ can be appreciated. In this case, it follows that the magnitude of $b$ can be retained to evaluate how strong is the increasing/decreasing trend of the dataset under study. Indeed, the MK null hypothesis is the absence of a trend in a dataset. Close-to- 0 values of $p_{M K}$ mean that the data behave consistently with the presence of a marked trend (i.e. the null hypothesis is rejected), and this is more likely to occur for high values of $b$, as shown in Figure 2 for the AM $H_{s}$ (similar considerations hold for annual $98^{\text {th }}$ percentile of $H_{s}$ and annual mean $H_{s}$ ). Furthermore, $b$ was proved to be correlated with the ITA outcomes. In fact, both the graphical analysis of the $\delta_{i}$ ecdf for the selected locations, and the correlation analysis of the sum of $\delta_{i}$ for all the hindcast locations, reveal a strong correlation of $\delta_{i}$ itself and $b$, in particular for the annual mean and maxima $H_{s}$ (as shown in Table 1). It follows that the $\delta_{i}$ identified by the ITA graphical method is in turn correlated to the $p_{M K}$. Therefore, this paper allows to use the information provided by $b$ to quantify trends, because of the correlation with $p_{M K}$, and it provides theoretical support to the ITA. The application here considered shows that the general use of $p_{M K}$, as recommended in [15], 
expands, with respect to the more usual usage of the MK, the knowledge of possible trends by attaching to each value of $b$ a measure of the consistency with the null hypothesis, without any a priori selection based on a threshold.

In view of the above, the values of $b$ were used to gain an insight into the spatial distribution of wave climate trends over the MS. In particular, the statistics employed in this work were selected as they can be of great importance in maritime and ocean engineering. The $\mathrm{AM} H_{s}$ are indicative of the most severe sea states, which are retained to compute the high return period distribution of $H_{s}$, to be further used in marine and coastal structural design. In the framework of Extreme Value Analysis, the $98^{\text {th }}$ percentile of the initial $H_{s}$ distribution of a sample is often used as a threshold to select the exceeding peaks in the POT approach. Finally, mean sea states can be relevant for fatigue analysis of maritime structures. The analysis revealed similar patterns among the spatial distributions of trends for the annual $98^{\text {th }}$ percentile of $H_{s}$ and annual mean $H_{s}$, while trends of AM $H_{s}$ are differently spread over the MS, moreover they are characterized by more intense values of $b$ (order of $\mathrm{cm} /$ year). These outcomes were then compared with previous researches aimed at detecting and computing trends over isolated spots in the MS. The order of magnitude of the annual rate of changes show good consistency with the values of $b$ computed in this work, while there are slight deviations in the sign of trends for some locations, as discussed in section 3.2. However, it has to be reminded that the exhaustively characterization of the wave climate trends in the MS is beyond the scope of this research, though interesting analogies with previous works can be pointed out and leave room for further investigation. 
Finally, it is worth mentioning that, although the paper focuses on sea states, the analysis here introduced can be extended to other parameters without loss of generality, and the application of this methodology to different geophysical time series is therefore straightforward.

\section{Acknowledgments}

The results for $b$ and $p_{M K}$ are available for download at Nottingham Research Data Management Repository, doi: 10.17639/nott.7016

\section{References}

[1] Adger, W.N., 1999. Social vulnerability to climate change and extremes in coastal Vietnam. World development 27, 249-269.

[2] Appendini, C.M., Torres-Freyermuth, A., Salles, P., López-González, J., Mendoza, E.T., 2014. Wave climate and trends for the gulf of mexico: A 30-yr wave hindcast. Journal of Climate 27, 1619-1632.

[3] Besio, G., Briganti, R., Romano, A., Mentaschi, L., De Girolamo, P., 2017. Time clustering of wave storms in the mediterranean sea. Natural Hazards and Earth System Sciences 17, 505-514.

[4] Cai, W., Cowan, T., 2007. Trends in Southern Hemisphere circulation in ipcc AR4 models over 1950-99: Ozone depletion versus greenhouse forcing. Journal of Climate 20, 681-693.

[5] Cai, W., Shi, G., Cowan, T., Bi, D., Ribbe, J., 2005. The response of the Southern Annular Mode, the East Australian Current, and the 
southern mid-latitude ocean circulation to global warming. Geophysical Research Letters 32.

[6] Casas-Prat, M., Sierra Pedrico, J.P., 2010. Trend analysis of the wave storminess: the wave direction. Advances in Geosciences 26, 89-92.

[7] Casas Prat, M., Sierra Pedrico, J.P., 2010. Trend analysis of wave storminess: wave direction and its impact on harbour agitation. Natural Hazards and Earth System Sciences 10, 2327-2340.

[8] Castelle, B., Dodet, G., Masselink, G., Scott, T., 2018. Increased wintermean wave height, variability, and periodicity in the northeast atlantic over 1949-2017. Geophysical Research Letters 45, 3586-3596.

[9] De Leo, F., Besio, G., Zolezzi, G., Bezzi, M., 2019. Coastal vulnerability assessment: through regional to local downscaling of wave characteristics along the bay of lalzit (albania). Natural Hazards and Earth System Sciences 19, 287-298.

[10] De Michele, C., Salvadori, G., Passoni, G., Vezzoli, R., 2007. A multivariate model of sea storms using copulas. Coastal Engineering 54, $734-751$.

[11] Dolan, A.H., Walker, I.J., 2006. Understanding vulnerability of coastal communities to climate change related risks. Journal of Coastal Research , 1316-1323.

[12] Doney, S.C., Ruckelshaus, M., Emmett Duffy, J., Barry, J.P., Chan, F., English, C.A., Galindo, H.M., Grebmeier, J.M., Hollowed, A.B., Knowl- 
ton, N., et al., 2012. Climate change impacts on marine ecosystems. Annual Review of Marine Science 4, 11-37.

[13] Durack, P.J., Wijffels, S.E., 2010. Fifty-year trends in global ocean salinities and their relationship to broad-scale warming. Journal of Climate $23,4342-4362$.

[14] Genest, C., Favre, A.C., 2007. Everything you always wanted to know about copula modeling but were afraid to ask. Journal of Hydrologic Engineering 12, 347-368.

[15] Greenland, S., Senn, S.J., Rothman, K.J., Carlin, J.B., Poole, C., Goodman, S.N., Altman, D.G., 2016. Statistical tests, P values, confidence intervals, and power: a guide to misinterpretations. European Journal of Epidemiology 31, 337-350.

[16] Gulev, S.K., Grigorieva, V., 2004. Last century changes in ocean wind wave height from global visual wave data. Geophysical Research Letters 31.

[17] Hamm, L., Capobianco, M., Dette, H., Lechuga, A., Spanhoff, R., Stive, M., 2002. A summary of European experience with shore nourishment. Coastal Engineering 47, 237-264.

[18] Harley, C.D., Randall Hughes, A., Hultgren, K.M., Miner, B.G., Sorte, C.J., Thornber, C.S., Rodriguez, L.F., Tomanek, L., Williams, S.L., 2006. The impacts of climate change in coastal marine systems. Ecology Letters 9, 228-241. 
[19] Kendall, M.G., 1955. Rank correlation methods .

[20] Koetse, M.J., Rietveld, P., 2009. The impact of climate change and weather on transport: An overview of empirical findings. Transportation Research Part D: Transport and Environment 14, 205-221.

[21] Laface, V., Malara, G., Romolo, A., Arena, F., 2016. Peak over threshold vis-à-vis equivalent triangular storm: Return value sensitivity to storm threshold. Coastal Engineering 116, 220-235.

[22] Liang, B., Shao, Z., Li, H., Shao, M., Lee, D., 2019. An automated threshold selection method based on the characteristic of extrapolated significant wave heights. Coastal Engineering 144, 22-32.

[23] Mann, H.B., 1945. Nonparametric tests against trend. Econometrica: Journal of the Econometric Society, 245-259.

[24] Mentaschi, L., Besio, G., Cassola, F., Mazzino, A., 2013. Developing and validating a forecast/hindcast system for the Mediterranean Sea. Journal of Coastal Research SI 65, 1551-1556.

[25] Mentaschi, L., Besio, G., Cassola, F., Mazzino, A., 2015. Performance evaluation of WavewatchIII in the Mediterranean Sea. Ocean Modelling 90, 82-94.

[26] Mori, N., Yasuda, T., Mase, H., Tom, T., Oku, Y., 2010. Projection of extreme wave climate change under global warming. Hydrological Research Letters 4, 15-19. 
[27] Morim, J., Hemer, M., Wang, X.L., Cartwright, N., Trenham, C., Semedo, A., Young, I., Bricheno, L., Camus, P., Casas-Prat, M., et al., 2019. Robustness and uncertainties in global multivariate wind-wave climate projections. Nature Climate Change 9, 711-718.

[28] Muraleedharan, G., Lucas, C., Soares, C.G., 2016. Regression quantile models for estimating trends in extreme significant wave heights. Ocean Engineering 118, 204-215.

[29] Musić, S., Nicković, S., 2008. 44-year wave hindcast for the Eastern Mediterranean. Coastal Engineering 55, 872-880.

[30] Nicholls, R.J., Cazenave, A., 2010. Sea-level rise and its impact on coastal zones. Science 328, 1517-1520.

[31] Piscopia, R., Inghilesi, R., Panizzo, A., Corsini, S., Franco, L., 2003. Analysis of 12-year wave measurements by the Italian Wave Network, in: Coastal Engineering 2002: Solving Coastal Conundrums. World Scientific, pp. 121-133.

[32] Pomaro, A., Cavaleri, L., Lionello, P., 2017. Climatology and trends of the Adriatic Sea wind waves: analysis of a 37-year long instrumental data set. International Journal of Climatology 37, 4237-4250.

[33] Richards, J., Mokrech, M., Berry, P., Nicholls, R., 2008. Regional assessment of climate change impacts on coastal and fluvial ecosystems and the scope for adaptation. Climatic Change 90, 141-167.

[34] Saha, S., Moorthi, S., Pan, H.L., Wu, X., Wang, J., Nadiga, S., Tripp, P., Kistler, R., Woollen, J., Behringer, D., Liu, H., Stokes, D., Grumbine, 
R., Gayno, G., Wang, J., Hou, Y.T., Chuang, H.Y., Juang, H.M.H., Sela, J., Iredell, M., Treadon, R., Kleist, D., Van Delst, P., Keyser, D., Derber, J., Ek, M., Meng, J., Wei, H., Yang, R., Lord, S., Van Den Dool, H., Kumar, A., Wang, W., Long, C., Chelliah, M., Xue, Y., Huang, B., Schemm, J.K., Ebisuzaki, W., Lin, R., Xie, P., Chen, M., Zhou, S., Higgins, W., Zou, C.Z., Liu, Q., Chen, Y., Han, Y., Cucurull, L., Reynolds, R.W., Rutledge, G., Goldberg, M., 2010. The NCEP climate forecast system reanalysis. Bulletin of the American Meteorological Society 91, 1015-1057.

[35] Scavia, D., Field, J.C., Boesch, D.F., Buddemeier, R.W., Burkett, V., Cayan, D.R., Fogarty, M., Harwell, M.A., Howarth, R.W., Mason, C., et al., 2002. Climate change impacts on US coastal and marine ecosystems. Estuaries 25, 149-164.

[36] Sen, P.K., 1968. Estimates of the regression coefficient based on kendall's tau. Journal of the American Statistical Association 63, 1379-1389.

[37] Şen, Z., 2011. Innovative trend analysis methodology. Journal of Hydrologic Engineering 17, 1042-1046.

[38] Şen, Z., 2013. Trend identification simulation and application. Journal of Hydrologic Engineering 19, 635-642.

[39] Shanas, P., Kumar, V.S., 2015. Trends in surface wind speed and significant wave height as revealed by era-interim wind wave hindcast in the central bay of bengal. International Journal of Climatology 35, 26542663. 
[40] Shi, J., Zheng, J., Zhang, C., Joly, A., Zhang, W., Xu, P., Sui, T., Chen, T., 2019. A 39-year high resolution wave hindcast for the chinese coast: Model validation and wave climate analysis. Ocean Engineering 183, $224-235$.

[41] Skamarock, W., Klemp, J., Dudhia, J., Gill, D., Barker, D., Wang, W., Powers, J., 2008. A Description of the Advanced Research WRF Version 3. Technical Note TN-468+STR. 113 pp. NCAR.

[42] Solari, S., Egüen, M., Polo, M.J., Losada, M.A., 2017. Peaks over threshold (POT): A methodology for automatic threshold estimation using goodness of fit p-value. Water Resources Research 53, 2833-2849.

[43] Stive, M.J., 2004. How important is global warming for coastal erosion? Climatic Change 64, 27-39.

[44] Theil, H., 1992. A rank-invariant method of linear and polynomial regression analysis, in: Henri Theil's Contributions to Economics and Econometrics. Springer, pp. 345-381.

[45] Tolman, H.L., 2009. User manual and system documentation of WAVEWATCH III version 3.14. Technical Report. NOAA/NWS/NCEP/MMAB.

[46] Vanem, E., 2016. Joint statistical models for significant wave height and wave period in a changing climate. Marine Structures 49, 180-205.

[47] Vanem, E., Walker, S.E., 2013. Identifying trends in the ocean wave climate by time series analyses of significant wave heightdata. Ocean Engineering 61, 148-160. 
538 [48] Wasserstein, R.L., Lazar, N.A., et al., 2016. The ASAs statement on 539 p-values: context, process, and purpose. The American Statistician 70, $540 \quad 129-133$.

${ }_{541}$ [49] Wdowinski, S., Bray, R., Kirtman, B.P., Wu, Z., 2016. Increasing flood542 ing hazard in coastal communities due to rising sea level: Case study of 543 miami beach, florida. Ocean \& Coastal Management 126, 1-8.

544 [50] Weisse, R., 2010. Marine Climate and climate Change: Storms, Wind $545 \quad$ Waves and Storm Surges. Springer Science \& Business Media.

546 [51] Young, I.R., Ribal, A., 2019. Multiplatform evaluation of global trends ${ }_{547}$ in wind speed and wave height. Science 364, 548-552. 